\title{
5-HTTLPR Biases Amygdala Activity in Response to Masked Facial Expressions in Major Depression
}

\author{
Udo Dannlowski, ${ }^{1,2}$, Patricia Ohrmann', Jochen Bauer', Jürgen Deckert ${ }^{1,3}$, Christa Hohoff', Harald Kugel ${ }^{4}$, \\ Volker Arolt', Walter Heindel ${ }^{4}$, Anette Kersting', Bernhard T Baune ${ }^{1,5}$ and Thomas Suslow*,I \\ 'Department of Psychiatry, University of Münster, Münster, Germany; ${ }^{2}$ IZKF-Research Group 4, IZKF Münster, University of Münster, Münster, \\ Germany; ${ }^{3}$ Department of Psychiatry, University of Würzburg, Würzburg, Germany; ${ }^{4}$ Department of Clinical Radiology, University of Münster, \\ Münster, Germany; ${ }^{5}$ Department of Psychiatry, James Cook University, Townsville, QLD, Australia
}

\begin{abstract}
The amygdala is a key structure in a limbic circuit involved in the rapid and unconscious processing of facial emotions. Increased amygdala reactivity has been discussed in the context of major depression. Recent studies reported that amygdala activity during conscious emotion processing is modulated by a functional polymorphism in the serotonin transporter gene (5-HTTLPR) in healthy subjects. In the present study, amygdala reactivity to displays of emotional faces was measured by means of $\mathrm{fMRI}$ at $3 \mathrm{~T}$ in 35 patients with major depression and 32 healthy controls. Conscious awareness of the emotional stimuli was prevented via backward-masking to investigate automatic emotion processing. All subjects were genotyped for the 5 -HTTLPR polymorphism. Risk allele carriers $\left(S\right.$ or $\left.L_{G}\right)$ demonstrated increased amygdala reactivity to masked emotional faces, which in turn was significantly correlated with life-time psychiatric hospitalization as an index of chronicity. This might indicate that genetic variations of the serotonin transporter could increase the risk for depression chronification via altering limbic neural activity on a preattentive level of emotion processing.

Neuropsychopharmacology (2008) 33, 4I8-424; doi:I0.1038/sj.npp. I3014II; published online 4 April 2007
\end{abstract}

Keywords: genetics; fMRI; amygdala; SLC6A4; 5-HTTLPR; major depression

\section{INTRODUCTION}

Converging evidence suggests that the serotonin system plays a critical role in the pathophysiology of major depression (Wong and Licinio, 2001). During the last decade, genetic variations in the serotonin system have been discovered that might constitute susceptibility factors for the development of depression. The serotonin transporter (5-HTT) facilitates re-uptake of serotonin from the synaptic cleft and is the target of most antidepressant drugs. In the promoter region of the serotonin transporter gene, mapping to chromosome 17q11.1-12, a functional variable repeat sequence polymorphism (5-HTTLPR) resulting in a short (S) and a long (L) variant has been identified (Lesch et al, 1996). It was repeatedly found that $S$ allele carriers are more prone to the depressogenic effect of stressful life events than homozygotes for the L allele (Caspi et al, 2003; Kendler et al, 2005). Furthermore, 5-HTTLPR 'risk alleles' were reported to be associated with depression severity (Zalsman et al, 2006) and reduced therapy response (Smits et al, 2004). However, other studies failed to find associations of 5-

*Correspondence: Dr T Suslow, Department of Psychiatry, University of Münster, Albert-Schweitzer-Strasse II, 48149 Münster, Germany, Tel: + 492518356615 , Fax: + 492518356612 ,

E-mail: Thomas.Suslow@ukmuenster.de

Received I5 November 2006; revised 2 March 2007; accepted 5 March 2007
HTTLPR with major depression (Willis-Owen et al, 2005). We investigated 5-HTTLPR in combination with a recently discovered single nucleotide polymorphism (SNP) with an A-G substitution ( $r$ 25531). Since the $S_{G}$ allele seems to be a rare variant (Wendland et al, 2006; and was not found in the present sample at all) several previous reports treated 5HTTLPR/rs25531 as a triallelic polymorphism. The $\mathrm{L}_{\mathrm{G}}$ allele was reported to behave comparable to the low expressing $\mathrm{S}$ allele (Hu et al, 2005). Furthermore, a recent PET study demonstrated in vivo that human $\mathrm{L}_{\mathrm{A}} / \mathrm{L}_{\mathrm{A}}$ carriers show higher 5-HTT binding potential in the putamen as an index of 5-HTT density (Praschak-Rieder et al, in press).

Associations of single polymorphisms with clinically defined phenotypes suffer from methodological difficulties (Malhotra and Goldman, 1999) and the heterogeneity and complexity of clinical phenotypes necessitate large sample sizes to detect rather small effects. A strategy to overcome such methodological problems is the endophenotype approach (Hasler et al, 2004). Hariri et al (2006) suggested that the examination of regional brain activation during emotion processing represents an innovative approach to link neural dysfunction to genes involved in the pathogenesis of depression with effect sizes 10-20 times larger than in classical association studies. The amygdala as a central processor of emotional significance has most intensively been studied in this emerging field called 'imaging genetics'. Increased amygdala reactivity is a frequent finding in major depression (eg Sheline et al, 
2001; Siegle et al, 2007) and a potential neural substrate of altered emotion processing in patients suffering from depression (Whalen et al, 2002). Several studies have demonstrated that healthy $S$ allele carriers show increased amygdala reactivity to emotional stimuli (Bertolino et al, 2005; Hariri et al, 2002, 2005; Heinz et al, 2005, Pezawas et al, 2005). Thus, it was speculated that the $S$ allele could increase the risk for depression via alterations of amygdala activity during emotion processing (Hariri et al, 2005). In a recent study, we have demonstrated that depressed patients carrying 5-HTTLPR risk alleles also show increased amygdala activity compared with depressed non-risk allele carriers (Dannlowski et al, in press).

However, all previous studies reporting influence of genetic variations on brain activity have employed paradigms using overt stimulus presentation with conscious processing of emotional stimuli. It remains unclear if the 5HTTLPR effect on amygdala activity is restricted to the conscious level of emotion processing or if it can already be found at an automatic, pre-attentive stage. According to neurobiological theories of emotions, the amygdala is particularly implicated in the rapid and automatic processing of emotional significance preceding conscious awareness (LeDoux, 1996). In healthy subjects, several studies have confirmed that the amygdala is engaged during processing of emotional stimuli, even if presented briefly $(<40 \mathrm{~ms})$ and backward-masked, and thus without conscious awareness (eg Whalen et al, 1998). Amygdala reactivity to covertly but not to overtly presented fear faces was associated with individual differences in trait anxiety (Etkin et al, 2004). Thus, using covert stimulus presentation might be a more appropriate approach to investigate the role of the amygdala in dispositional emotional reactivity. So far, however, there has not been any report of a genetic effect on neural activity to masked emotional stimuli neither in healthy controls nor in patients with depression.

In the present study, we therefore have investigated amygdala activity in response to masked displays of emotional faces in relation to the 5-HTTLPR genotype in both, a sample of depressed inpatients and healthy subjects. Success of the masking procedure was assessed by means of a forced choice detection task. We speculated that 5HTTLPR confers a change in amygdala activity at a preattentive stage of emotion processing, at which no conscious processing of the emotional faces has taken place.

\section{PATIENTS AND METHODS}

\section{Subjects}

Datasets of 35 inpatients with major depression, diagnosed with the SCID-I interview (Wittchen et al, 1997), and 32 healthy subjects were analyzed. Sociodemographic and clinical details of the study groups are shown in Table 1. Exclusion criteria were neurological abnormalities, substance abuse, former electroconvulsive therapy, age of 60 and above, and benzodiazepine treatment. Healthy subjects had no history of psychiatric disorders or psychotropic medication. All patients were under antidepressant medication, which was coded in terms of dose and treatment duration into medication levels from 1 to 4 , according to the suggestions of Sackeim (2001). Details of the antidepressant medication are listed in Table 2. No patient received lithium or anti-epileptic drugs. Two patients (both $\mathrm{S} / \mathrm{L}_{\mathrm{A}}$ genotype) received additional antipsychotic drugs (amisulprid and quetiapine). Excluding these two patients would not change the results. The experiments were conducted in accordance with the Declaration of Helsinki. Approval was obtained from the ethics committee at the University of Münster. After a comprehensive description of the study to the subjects, written informed consent was obtained.

\section{Genotyping}

All subjects were genotyped for the 5-HTTLPR polymorphism, including SNP rs25531 (A/G), according to published protocols (Deckert et al, 1997; Wendland et al, 2006) with minor variation. Primers 5'-GGCGTTGCCGCTCTGAATGC$3^{\prime}$ and $5^{\prime}$-GAGGGACTGAGCTGGACAACCAC- $3^{\prime}$ (10 pM each) were used for $20 \mu \mathrm{l}$ PCR containing $60 \mathrm{ng}$ DNA, $200 \mu \mathrm{M}$ dNTPs, $\mathrm{H}_{2} \mathrm{O}$ and $0.5 \mathrm{U}$ HotStar Taq Polymerase with $1.5 \mathrm{mM} \mathrm{MgCl}_{2}, 1 \times$ Q-Solution and $1 \times$ Buffer (Qiagen), with an initial $15 \mathrm{~min}$ denaturation step at $95^{\circ} \mathrm{C}$ followed by 35 PCR cycles of $94^{\circ} \mathrm{C}(60 \mathrm{~s}), 64^{\circ} \mathrm{C}(60 \mathrm{~s})$ and $72^{\circ} \mathrm{C}(120 \mathrm{~s})$ and a final extension step of $10 \mathrm{~min}$ at $72^{\circ} \mathrm{C}$. For RFLP analysis PCR products were digested with $\mathrm{HpaII}$ at $37^{\circ} \mathrm{C}$ overnight, separated in $15 \%$ polyacrylamide gels $(1 \times \mathrm{TBE}, 230 \mathrm{~V} / \mathrm{cm})$ for $3.5 \mathrm{~h}$ and visualized by silver staining, which resulted in fragments between 62 and $340 \mathrm{bp}$ length allowing differentiation and assignment of all 5-HTT-LPR and -rs25531 genotypes. For genotyping quality control about one-third

Table I Clinical and Affective Characteristics of Study Participants after Exclusion of Subjects Performing Above Chance in the Detection Task

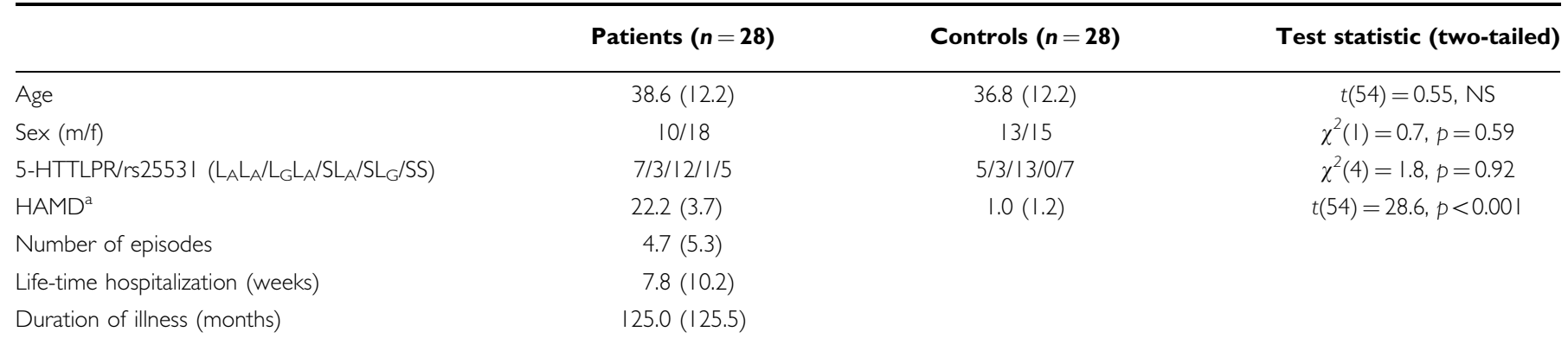

aHAMD, Hamilton Rating Scale for Depression (Hamilton, 1960). 
Table 2 List of Antidepressant Medication in the Genotype Groups

5-HTTLPR/5-HT-rs2553 I

\begin{tabular}{lcc}
\cline { 2 - 3 } & $\mathbf{L}_{\mathbf{A}} \mathbf{L}_{\mathbf{A}}(\mathbf{n}=\mathbf{7})$ & $\mathbf{S S} / \mathbf{S L} / \mathbf{L}_{\mathbf{A}} \mathbf{L}_{\mathbf{G}}(\boldsymbol{n}=\mathbf{2} \mathbf{I})$ \\
\hline Venlafaxine & 3 & 5 \\
Venlafaxine+Mirtazapine & $\mathrm{I}$ & 3 \\
Escitalopram & $\mathrm{I}$ & 5 \\
Escitalopram+Mirtazapine & 0 & 2 \\
Duloxetine & 0 & 1 \\
Fluoxetine & 0 & 1 \\
Sertraline+Mirtazapine & 1 & 0 \\
Mirtazapine & 1 & 3 \\
Nortriptyline & 0 & 1
\end{tabular}

Values reflect number of patients treated with the respective medication.

of probands were additionally genotyped by direct automated sequencing, which resulted in concordance rates of $100 \%$. The genotype distribution of the 5-HTTLPR did not differ significantly from the expected numbers calculated according to the Hardy-Weinberg equilibrium. Following previous studies, subjects were grouped into risk (S) allele carriers and non-risk $\left(\mathrm{L}_{\mathrm{A}}\right)$ allele carriers for each polymorphism. Since the $\mathrm{L}_{\mathrm{G}}$ and $\mathrm{S}$ alleles have comparable levels of serotonin transporter expression ( $\mathrm{Hu}$ et al, 2005), the $\mathrm{L}_{\mathrm{G}}$ allele was treated as risk allele for the purpose of grouping. See Table 1, for a description of allele frequencies. According to $t$-tests or $\chi^{2}$-tests, risk allele carriers and non-risk allele carriers were not significantly different concerning age, gender, education, verbal intelligence, and, for patients, medication level, medication type, depression severity, duration of illness, number of episodes or total hospitalization time (all $p>0.25$ ).

\section{fMRI Methods}

Technical details of the fMRI data acquisition and processing have been reported (Domschke et al, 2006). Briefly, facial stimuli consisted of sad, angry, happy and neutral expressions (Ekman and Friesen, 1976). Subjects were presented with alternating $30 \mathrm{~s}$ epochs of a face category or a no-face stimulus (a grey rectangle). In a passive viewing task facial stimuli were presented twice per second for $33 \mathrm{~ms}$ in a random sequence directly followed by a $467 \mathrm{~ms}$ mask depicting a neutral face of the same actor. Each emotion epoch was preceded by a no-face epoch and was presented twice, resulting in a total presentation time of $8 \mathrm{~min}$. T2* functional data were acquired at a $3 \mathrm{~T}$ scanner (Gyroscan Intera 3.0T, Philips Medical Systems, Best, NL) using a single shot echoplanar sequence with parameters selected to minimize distortion in the amygdala while retaining adequate $\mathrm{S} / \mathrm{N}$ and $\mathrm{T} 2 *$ sensitivity according to suggestions made by Robinson et al (2003). Volumes consisting of 25 axial slices were acquired (matrix $128^{\star} 128$, resolution $\left.1.75^{\star} 1.75^{\star} 3.5 \mathrm{~mm} ; \mathrm{TR}=3 \mathrm{~s}, \mathrm{TE}=30 \mathrm{~ms}, \mathrm{FA}=90^{\circ}\right) 160$ times in blocked design, 10 times per condition. To optimize the following normalization procedures, the same sequenceparameters were used to cover the whole brain with 43 slices. Additionally, two anatomical data sets were acquired: $\mathrm{T} 1$ weighted inversion recovery and a high resolution $\mathrm{T} 1$ weighted $3 \mathrm{D}$ sequence (isotropic voxel, $0.5 \mathrm{~mm}$ edge length).

Functional imaging data were motion corrected, using a set of six rigid body transformations determined for each image, spatially normalized to standard MNI space (Montreal Neurological Institute) with a voxel size of $2 * 2 * 2 \mathrm{~mm}$, and smoothed (Gaussian kernel, $6 \mathrm{~mm}$ FWHM) using Statistical Parametric Mapping (SPM2, Wellcome Department of Cognitive Neurology, London, UK). The first (individual) level analysis was performed by modeling the different conditions (angry, sad, happy, and neutral) as variables within the general linear model (modeled with a standard hemodynamic response function), contrasting emotional faces (angry, sad and happy) with the neutral face condition. Voxel values of bilateral amygdala were extracted, summarized by mean and tested among the different conditions using the MarsBaR toolbox (Brett et al, 2002). The bilateral amygdala mask was defined according to a published anatomical atlas, (Tzourio-Mazoyer et al, 2002) which comprised 448 voxels (ie $3.6 \mathrm{ml}$ ) and included the grey nuclei at the rostral boundary of the hippocampus and the caudal boundary of the uncus.

Repeated measures analysis of variance (ANOVA) was conducted on mean amygdala activation parameters (contrast values). Since left and right amygdala activations were highly inter-correlated in all three emotion conditions (all $r>0.74$ ), an averaged measure for bilateral amygdala activity was used.

To explore genotype effects in brain regions outside the amygdala, a voxel-wise approach was employed. The first level contrast images were included in exploratory second level group analyses for group and genotype effects. In addition to the amygdala, the anterior cingulate cortex (ACC) was treated as region of interest (ROI), since the ACC has been implicated in the processing of masked emotional faces in a previous study (Killgore and Yurgelun-Todd, 2004) and was found to activate depending on 5-HTTLR genotype during emotion processing (Pezawas et al, 2005). The WFU PickAtlas Toolbox (Maldjian et al, 2003) was used to create an amygdala/ACC ROI mask for small volume correction according to the definitions of Tzourio-Mazoyer et al (2002). The statistical threshold was set at $p<0.05$, corrected for the amygdala/ACC volume. For the genotype comparison within each of the two subgroups, a more lenient threshold of $p<0.01$, uncorrected was chosen. Outside these anatomical areas with strong a priori hypothesis, a threshold of $p<0.05$, corrected for the entire brain was employed.

\section{Detection Task}

The detection task was designed to assess possible objective awareness of the masked emotional faces. After the experiment, the subjects were informed about the presentation of covert emotional faces in the MRI scanner. Then they were asked for subjective awareness of the emotional prime faces. In the following, the detection task was administered outside the scanner. Each of the 40 trials had the following routine: after a fixation cross lasting for $500 \mathrm{~ms}$, a prime face was presented for $33 \mathrm{~ms}$ that was directly followed by a neutral target face. Each prime 

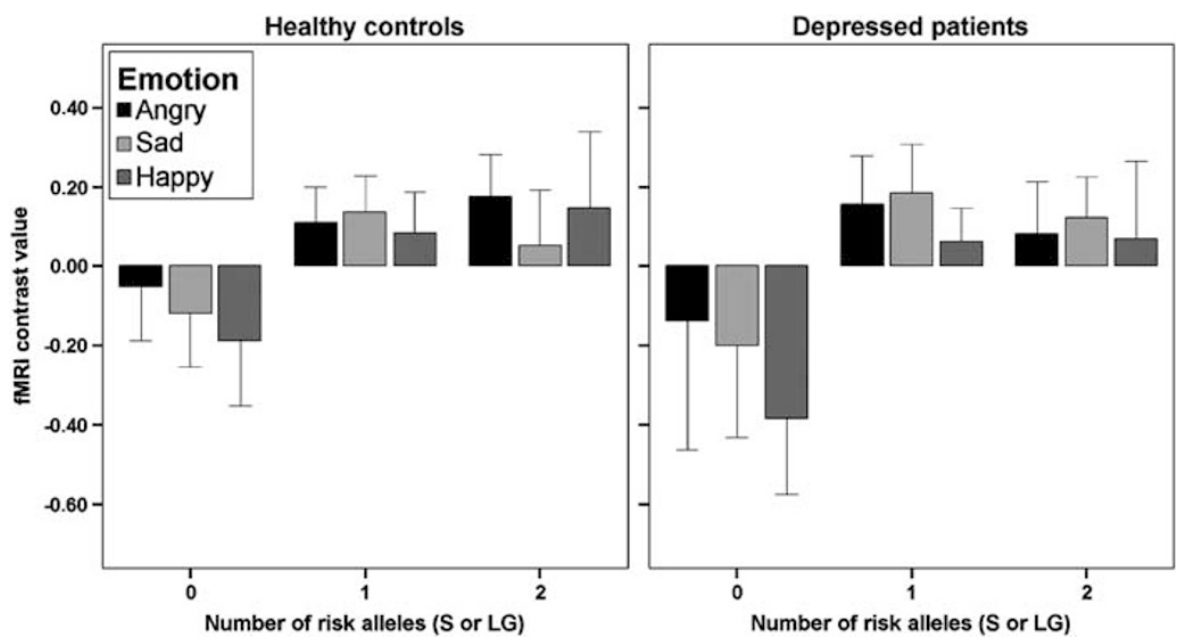

Figure I Bar graphs depicting mean amygdala activation to angry, sad and happy facial expressions in comparison to the neutral face condition dependent on group (patients vs controls) and number of risk alleles ( $\mathrm{S}$ or $\mathrm{L}_{\mathrm{G}}$ allele) in the 5-HTTLPR/5-HT-rs2553I polymorphisms.

emotion (angry, sad, happy and neutral) was presented 10 times and the order of trials was randomized for each subject. The instruction was to indicate which emotion quality was displayed briefly as prime. The chance level for correct answers was $25 \%$. Binominal tests were used to explore whether a subject performed above chance.

\section{RESULTS}

\section{Detection Task}

One healthy subject (genotype S/S) reported having consciously detected brief displays of emotional faces inside the scanner. This subject also performed above chance in the detection task $(45 \%, p=0.002)$. All other participants reported not having seen any masked emotional faces. However, three more healthy subjects $\left(\mathrm{S} / \mathrm{S}, \mathrm{S} / \mathrm{L}_{\mathrm{A}}\right.$, and $\left.\mathrm{L}_{\mathrm{A}} / \mathrm{L}_{\mathrm{A}}\right)$ and seven patients $\left(4 \mathrm{~S} / \mathrm{L}_{\mathrm{A}}, \mathrm{S} / \mathrm{S}, \mathrm{S} / \mathrm{L}_{\mathrm{G}}\right.$, and $\left.\mathrm{L}_{\mathrm{A}} / \mathrm{L}_{\mathrm{G}}\right)$ performed above chance in the detection task. These subjects were considered 'aware' and consequently they were removed from further analysis. The remaining subjects (28 patients and 28 controls) performed at or below chance level with no differences between patients and controls (mean hit rate $=26.4 \%$; range: $12.5-35 \%$ ). However, inclusion of the eliminated data would not change the pattern of results.

\section{fMRI Results}

Dependent variables were mean amygdala contrast values in response to masked angry, sad and happy faces versus neutral faces (Figure 1). A 3 (emotion type: angry, sad, happy) $\times 2$ (group: patients, controls) $\times 2$ (genotype: risk, non-risk) analysis of variance was conducted on the mean amygdala responses. Box's test indicated equality of covariance matrices (Box's $M=33.5, \mathrm{NS}$ ) and Mauchly's test yielded no violation of the sphericity assumption $(W=0.95, \mathrm{NS})$. A main effect of 5 -HTTLPR genotype was found, $\mathrm{F}(1,52)=6.2, p=0.016$, Cohen's $d=0.79$. As expected, risk allele carriers ( $S$ allele or $\mathrm{L}_{\mathrm{G}}$ allele carriers, $n=44)$ demonstrated increased amygdala activity compared with $\mathrm{L}_{\mathrm{A}} / \mathrm{L}_{\mathrm{A}}$ homozygotes $(n=12$, see Figure 2$)$. The

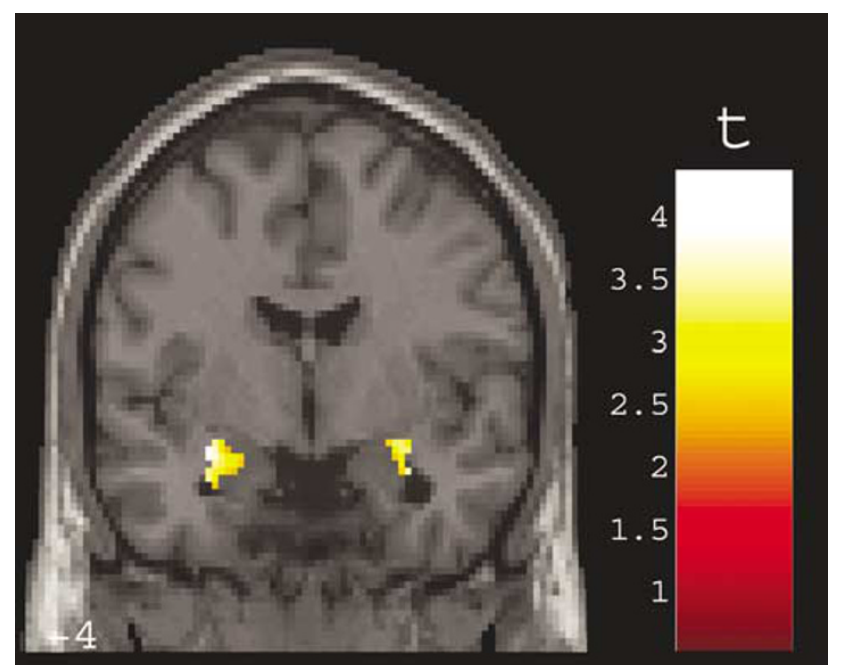

Figure 2 Coronal view $(y=-4)$ depicting the main effect of 5-HTTLPR/ 5-HT-rs2553I on amygdala reactivity. Amygdala reactivity differences are shown between risk allele carriers ( $S$ or $L_{G}$ allele, $n=44$ ) and non-risk allele carriers $\left(L_{A} / L_{A}, n=12\right)$ in response to masked negative faces (angry and sad vs neutral). Voxel threshold was set at $p<0.05$, with FDR (false discovery rate) correction for bilateral amygdala volume. Cluster maxima are located at $\mathrm{MNI}$ coordinates $x=-30, y=-4, z=-14$ (left amygdala, 92 voxels, $Z=3.60$, p corrected $=0.017$ ) and $x=30, y=-6, z=-14$ (right amygdala, 41 voxels, $Z=3.35$, $\left.p_{\text {corrected }}=0.024\right)$.

genotype effect was not modulated by group, $\mathrm{F}(1,52)=0.2$, $p=0.6$. No other main effect or interaction of emotion type or group was recorded, although a power analysis with G-Power (Erdfelder et al, 1996) indicated sufficient power (0.83) to detect 'medium' size interactions (Cohen, 1988). In addition, the 5-HTTLPR effect was also found in the patient group alone, $\mathrm{F}(1,26)=4.4, p=0.046$, Cohen's $d=0.92$. However, in the control group, amygdala activity differences between risk and non-risk allele carriers did not reach significance, $\mathrm{F}(1,26)=2.1, p=0.16$, Cohen's $d=0.71$.

The voxel-wise analysis of the genotype effect in the whole sample confirmed the ANOVA result of stronger amygdala responses in risk allele carriers [left amygdala: $k$ (cluster size) $=93$ voxels, peak voxel at $x=-30, y=-4$, 
$z=-14, Z=3.6, p_{\text {corrected }}=0.024$; right amygdala: $k=45$, $\left.x=30, \quad y=-6, \quad z=-14, \quad Z=3.55, \quad p_{\text {corrected }}=0.024\right]$. Furthermore, a large and highly significant cluster in the ACC was found $[k=854, x=4, y=16, z=26, Z=3.98$, $p_{\text {corrected }}=0.001$, extending from the supragenual ACC to the perigenual area, $x=-2, y=44, z=2]$. The genotype effect was also found in the patient group alone [left amygdala: $k=35$ voxels, $x=-30, y=-2, z=-22, Z=3.14$, $p_{\text {uncorrected }}=0.001$; right amygdala: $k=5, x=28, y=-4$, $z=-12, Z=2.61, p_{\text {uncorrected }}=0.007$; ACC: $k=91, x=-6$, $y=28, z=30, Z=2.88, p_{\text {uncorrected }}=0.002$, extending to $x=4, y=30, z=-2$ ] and in the healthy control group [right amygdala: $k=6, x=30, y=-8, z=-12, Z=2.50$, $p_{\text {uncorrected }}=0.006 ; \quad$ ACC: $k=175, \quad x=2, \quad y=14, \quad z=28$, $Z=3.48, p_{\text {uncorrected }}=0.001$, extending to $x=-2, y=50$, $z=2$ ]. No genotype effect was detected in brain areas without a specific a priori hypothesis.

An exploratory analysis of group differences yielded no significant results in the amygdala/ACC ROI or other brain areas.

\section{Allele Load}

A recent study reported a linear increase of amygdala responses to overt negative pictures with risk (S) allele load (Heinz et al, 2005). To explore whether a linear increase of amygdala activation is responsible for the observed risk $v s$ non-risk group effect, we conducted a voxel-wise linear regression analysis of amygdala responses to masked negative faces in the whole sample. Genotype was coded as the number of risk alleles ( $\mathrm{S}$ or $\mathrm{L}_{\mathrm{G}} ; 0,1$, or 2 ). Applying the same statistical threshold as in the group comparison $(p<0.05$, corrected for the amygdala/ACC ROI or the entire brain), no significant effect of allele load was detected, indicating a 'dominant' effect of risk allele carriage.

\section{Amygdala Reactivity and Clinical Characteristics}

Amygdala reactivity to any emotion quality was not significantly associated with number of episodes or duration of illness, although all correlations carried a positive sign. However, we observed significant associations of lifetime psychiatric hospitalization and amygdala reactivity to masked angry $(r=0.38, p=0.046)$, sad $(r=0.44, p=0.020)$ and happy $(r=0.40, p=0.037)$ expressions. Thus, patients showing increased amygdala reactivity had a history of longer previous psychiatric hospitalization. There was no significant association of amygdala reactivity and current depressive symptoms (HAMD-score, all $r<0.12$ ).

\section{Role of Medication}

To explore the role of antidepressant medication, patients were grouped into a low-dose group (medication level 1-2, $n=12$ ) and a high-dose group (medication level 3-4, $n=16$ ) (Surguladze et al, 2005). High-and low-dose group did not differ with respect to 5-HTTLPR risk group $\left(\chi^{2}(1)=0.0, p=1.0\right)$ and amygdala activity elicited by any masked emotion type (all $p>0.2$ ). Adding medication level as covariate did not alter the results.

\section{DISCUSSION}

The present data suggest that amygdala reactivity to emotional faces is modulated by the 5-HTTLPR polymorphism even in absence of conscious processing of the emotional stimuli. These findings replicate and extend the common observation in healthy subjects that 5-HTTLPR risk allele carriers demonstrate stronger amygdala activity in response to emotional faces and pictures (Bertolino et al, 2005; Hariri et al, 2002, 2005; Heinz et al, 2005; Pezawas et al, 2005). Recently, Smolka et al (2007) reported that the 5 -HTTLPR effect on amygdala and anterior cingulate cortex activity in response to unpleasant pictures is stronger if the triallelic variant was considered compared with the 5HTTLPR effect alone. However, the present study is the first imaging genetics investigation that used masked displays of facial expressions and examined patients with clinical depression. The biasing effect of 5-HTTLPR on amygdala activity was somewhat more pronounced in the patient group (although not significantly, as indicated by a nonsignificant genotype $\times$ group interaction). Furthermore, in this group, amygdala reactivity was associated with life-time psychiatric hospitalization, as an index of illness chronicity. Our data may thus suggest that 5-HTT genotype affects the course of major depression by biasing amygdala activity during automatic emotion processing.

Interestingly, the 5-HTTLPR effect was not restricted to negative (angry and sad) emotions. Masked happy expressions elicited stronger amygdala activity in risk allele carriers as well, a finding in line with a recent study in which amygdala activity in a happy face condition was found to be increased in $S$ allele carriers suffering from panic disorder (Domschke et al, 2006). In addition, the only study so far that investigated amygdala activity in response to masked facial expressions in patients suffering from major depression demonstrated that unmedicated patients show increased amygdala activity compared to controls also for masked happy faces (Sheline et al, 2001). It could be argued that increased amygdala activity in response to emotional faces might indicate negatively biased emotion processing regardless of the stimulus valence, even processing of happy facial expressions. This corresponds well with a recent masked affective priming study (Dannlowski et al, 2006). It was found that acutely depressed patients demonstrate automatic negative evaluative biases elicited by masked emotional faces, including happy expressions, which predicted weak therapy outcome. However, since no evaluation data were collected in the present experiment this interpretation remains speculative. An alternative explanation of the observed 5-HTTLPR effect might be that risk allele carriers are in general more sensitive in the automatic detection of biologically and socially relevant information in the environment. There is substantial evidence that the human amygdala is involved in the appraisal of events relevant for the survival and well-being of the organism (Öhman, 2002; Sander et al, 2003).

According to our data 5-HTTLPR risk allele carriers show also an enhanced responsivity of the anterior cingulate cortex to masked facial emotions. The ventral ACC appears to have an important role in the production of affective states (Phillips et al, 2003) and in the detection of socially relevant visual stimuli encountered below the threshold of 
conscious perception (Killgore and Yurgelun-Todd, 2004). Thus, it can be hypothesized that risk allele carriers might be more affectively responsive to facial emotions and bring potentially important stimuli more easily into the forefront of conscious awareness.

Certain limitations must be acknowledged. Although the combined sample provided sufficient power to detect main effects of genotype or interactions, the number of non-risk allele carriers was small within the two subgroups, limiting the statistical power to detect genotype effects in the two groups considered alone. No follow-up data were assessed and the individual history of stressful life events was not recorded. Thus, any conclusion with respect to a prognostic value or causal relationships of genetic susceptibility, amygdala reactivity and chronification remains speculative. The correlation analysis of amygdala reactivity and clinical characteristics was explorative and would not survive alpha correction for multiple comparisons. Furthermore, we did not find differences in amygdala activity between depressed patients and healthy controls. However, all of our patients were medicated. Hence, even in case of significant group differences it could not have been concluded whether any observed group effect was due to depression or a confounding effect of medication. Nonetheless, our data are in line with the findings of Sheline et al (2001) and Fu et al (2004). These previous studies found no differences between medicated patients and healthy controls with respect to their amygdala reactivity to emotional faces. Although we did not find any effect of current antidepressant medication level on amygdala reactivity or any clinical characteristic in our patient group, the present data cannot necessarily be generalized to unmedicated patients. Further studies should address these issues by investigating unmedicated patients in longitudinal designs.

In summary, our preliminary findings provide evidence that genetic susceptibility factors for major depression might be transported via altered limbic neural activity already at a preattentive level of emotion processing. The findings of the present study hopefully stimulate further investigations of depressed patients employing the imaging genetics approach. The consistency of previous studies and the present data underscore the power of direct assessment of regional brain activity in exploring the functional impact of genetic variation in clinical states.

\section{ACKNOWLEDGEMENTS}

This study was supported by an IMF grant (AR 510403) by the Medical Faculty of the University of Münster.

\section{REFERENCES}

Bertolino A, Arciero G, Rubino V, Latorre V, De Candia M, Mazzola V et al (2005). Variation of human amygdala response during threatening stimuli as a function of 5'HTTLPR genotype and personality style. Biol Psychiatry 57: 1517-1525.

Brett M, Anton JL, Valabregue R, Poline JB. Region of interest analysis using an SPM toolbox [abstract]. Presented at the 8th International Conference on Functional Mapping of the Human Brain, June 2-6, 2002, Sendai, Japan. Available on CD-ROM in NeuroImage, Vol 16, No. 2, abstract 497.
Caspi A, Sugden K, Moffitt TE, Taylor A, Craig IW, Harrington H et al (2003). Influence of life stress on depression: moderation by a polymorphism in the 5-HTT gene. Science 301: 386-389.

Cohen J (1988). Statistical Power Analysis for the Behavioral Sciences. Erlbaum: Hillsdale, NJ, USA.

Dannlowski U, Kersting A, Donges U, Lalee-Mentzel J, Arolt V, Suslow T (2006). Subliminal facial affect priming is associated with therapy response in clinical depression. Eur Arch Psychiatry Clin Neurosci 256: 215-221.

Dannlowski U, Ohrmann P, Bauer J, Kugel H, Baune BT, Hohoff C et al Serotonergic genes modulate amygdala activity in major depression. Genes Brain Behav (in press).

Deckert J, Catalano M, Heils A, Di Bella D, Friess F, Politi E et al (1997). Functional promoter polymorphism of the human serotonin transporter: lack of association with panic disorder. Psychiatr Genet 7: 45-47.

Domschke K, Braun M, Ohrmann P, Suslow T, Kugel H, Bauer J et al (2006). Association of the functional $-1019 \mathrm{C} / \mathrm{G} 5-\mathrm{HT} 1 \mathrm{~A}$ polymorphism with prefrontal cortex and amygdala activation measured with 3T fMRI in panic disorder. Int J Neuropsychopharmacol 9: 349-355.

Ekman P, Friesen WV (1976). Pictures of Facial Affect. Consulting Psychologists Press: Palo Alto, CA, USA.

Erdfelder E, Faul F, Buchner A (1996). GPOWER: A general power analysis program. Behav Res Methods Instr Comp 28: 1-11.

Etkin A, Klemenhagen KC, Dudman JT, Rogan MT, Hen R, Kandel ER et al (2004). Individual differences in trait anxiety predict the response of the basolateral amygdala to unconsciously processed fearful faces. Neuron 44: 1043-1055.

Fu CH, Williams SC, Cleare AJ, Brammer MJ, Walsh ND, Kim J et al (2004). Attenuation of the neural response to sad faces in major depression by antidepressant treatment: a prospective, event-related functional magnetic resonance imaging study. Arch Gen Psychiatry 61: 877-889.

Hamilton M (1960). A rating scale for depression. J Neurol Neurosurg Psychiatry 23: 56-62.

Hariri AR, Drabant EM, Munoz KE, Kolachana BS, Mattay VS, Egan MF et al (2005). A susceptibility gene for affective disorders and the response of the human amygdala. Arch Gen Psychiatry 62: $146-152$.

Hariri AR, Drabant EM, Weinberger DR (2006). Imaging genetics: perspectives from studies of genetically driven variation in serotonin function and corticolimbic affective processing. Biol Psychiatry 59: 888-897.

Hariri AR, Mattay VS, Tessitore A, Kolachana B, Fera F, Goldman $\mathrm{D}$ et al (2002). Serotonin transporter genetic variation and the response of the human amygdala. Science 297: 400-403.

Hasler G, Drevets WC, Manji HK, Charney DS (2004). Discovering endophenotypes for major depression. Neuropsychopharmacology 29: 1765-1781.

Heinz A, Braus DF, Smolka MN, Wrase J, Puls I, Hermann D et al (2005). Amygdala-prefrontal coupling depends on a genetic variation of the serotonin transporter. Nat Neurosci 8: 20-21.

$\mathrm{Hu}$ X, Oroszi G, Chun J, Smith TL, Goldman D, Schuckit MA (2005). An expanded evaluation of the relationship of four alleles to the level of response to alcohol and the alcoholism risk. Alcohol Clin Exp Res 29: 8-16.

Kendler KS, Kuhn JW, Vittum J, Prescott CA, Riley B (2005). The interaction of stressful life events and a serotonin transporter polymorphism in the prediction of episodes of major depression: a replication. Arch Gen Psychiatry 62: 529-535.

Killgore WD, Yurgelun-Todd DA (2004). Activation of the amygdala and anterior cingulate during nonconscious processing of sad versus happy faces. Neuroimage 21: 1215-1223.

LeDoux J (1996). The Emotional Brain. The Mysterious Underpinnings of Emotional Life. Simon and Schuster: New York.

Lesch KP, Bengel D, Heils A, Sabol SZ, Greenberg BD, Petri S et al (1996). Association of anxiety-related traits with a 
polymorphism in the serotonin transporter gene regulatory region. Science 274: 1527-1531.

Maldjian JA, Laurienti PJ, Kraft RA, Burdette JH (2003). An automated method for neuroanatomic and cytoarchitectonic atlas-based interrogation of fMRI data sets. Neuroimage 19: 1233-1239.

Malhotra AK, Goldman D (1999). Benefits and pitfalls encountered in psychiatric genetic association studies. Biol Psychiatry 45: 544-550.

Öhman A (2002). Automaticity and the amygdala: nonconscious responses to emotional faces. Curr Dir Psychol Sci 11: 62-66.

Pezawas L, Meyer-Lindenberg A, Drabant EM, Verchinski BA, Munoz KE, Kolachana BS et al. (2005). 5-HTTLPR polymorphism impacts human cingulate-amygdala interactions: a genetic susceptibility mechanism for depression. Nat Neurosci 8: $828-834$.

Praschak-Rieder N, Kennedy J, Wilson AA, Hussey D, Boovariwala A, Willeit $\mathrm{M}$ et al Novel 5-HTTLPR Allele Associates with Higher Serotonin Transporter Binding in Putamen: A [(11)C] DASB Positron Emission Tomography Study. Biol Psychiatry (in press).

Phillips ML, Drevets WC, Rauch SL, Lane R (2003). Neurobiology of emotion perception I: the neural basis of normal emotion perception. Biol Psychiatry 54: 504-514.

Robinson S, Windischberger C, Rauscher A, Moser E (2003). Optimized 3T EPI of the amygdala. NeuroImage 22: 203-210.

Sackeim HA (2001). The definition and meaning of treatmentresistant depression. J Clin Psychiatry 62: S1-S17.

Sander D, Grafman J, Zalla T (2003). The human amygdala: an evolved system for relevance detection. Rev Neurosci 14: 303-316.

Sheline YM, Barch DM, Donnelly JM, Ollinger JM, Snyder AZ, Mintun MA (2001). Increased amygdala response to masked emotional faces in depressed subjects resolves with antidepressant treatment: an fMRI study. Biol Psychiatry 50: 651-658.

Siegle GJ, Thompson W, Carter CS, Steinhauer SR, Thase ME (2007). Increased amygdala and decreased dorsolateral prefrontal BOLD responses in unipolar depression. Related and independent features. Biol Psychiatry 61: 198-209.

Smits KM, Smits LJ, Schouten JS, Stelma FF, Nelemans P, Prins MH (2004). Influence of SERTPR and STin2 in the serotonin transporter gene on the effect of selective serotonin reuptake inhibitors in depression: a systematic review. Mol Psychiatry 9: 433-441.

Smolka MN, Buhler M, Schumann G, Klein S, Hu XZ, Moayer M et al (2007). Gene-gene effects on central processing of aversive stimuli. Mol Psychiatry 12: 307-317.

Surguladze S, Brammer MJ, Keedwell P, Giampietro V, Young AW, Travis MJ et al (2005). A differential pattern of neural response toward sad versus happy facial expressions in major depressive disorder. Biol Psychiatry 57: 201-209.

Tzourio-Mazoyer N, Landeau B, Papathanassiou D, Crivello F, Etard O, Delcroix N et al (2002). Automated anatomical labeling of activations in SPM using a macroscopic anatomical parcellation of the MNI MRI single-subject brain. NeuroImage 15: 273-289.

Wendland JR, Martin BJ, Kruse MR, Lesch KP, Murphy DL (2006). Simultaneous genotyping of four functional loci of human SLC6A4, with a reappraisal of 5-HTTLPR and rs25531. Mol Psychiatry 11: 224-226.

Whalen PJ, Rauch SL, Etcoff NL, McInerney SC, Lee MB, Jenike MA (1998). Masked presentations of emotional facial expressions modulate amygdala activity without explicit knowledge. J Neurosci 18: 411-418.

Whalen PJ, Shin LM, Somerville LH, McLean AA, Kim H (2002). Functional neuroimaging studies of the amygdala in depression. Semin Clin Neuropsychiatry 7: 234-242.

Willis-Owen SA, Turri MG, Munafo MR, Surtees PG, Wainwright NW, Brixey RD et al (2005). The serotonin transporter length polymorphism, neuroticism, and depression: a comprehensive assessment of association. Biol Psychiatry 58: 451-456.

Wittchen HU, Wunderlich U, Gruschwitz S, Zaudig M (1997). SKID-I, Strukturiertes Klinisches Interview für DSM-IV. Hogrefe: Göttingen, Germany.

Wong ML, Licinio J (2001). Research and treatment approaches to depression. Nat Rev Neurosci 2: 343-351.

Zalsman G, Huang YY, Oquendo MA, Burke AK, Hu XZ, Brent DA et al (2006). Association of a triallelic serotonin transporter gene promoter region (5-HTTLPR) polymorphism with stressful life events and severity of depression. Am J Psychiatry 163: 1588-1593. 